\title{
MODIS Solar Reflective Calibration Traceability
}

\author{
Xiaoxiong (Jack) Xiong and Jim. Butler \\ Sciences and Exploration Directorate, NASA/GSFC, Greenbelt, MD 20771, USA \\ Xiaoxiong.Xiong-1@nasa.gov
}

\begin{abstract}
Long-term climate data records often consist of observations made by multiple sensors. It is, therefore, extremely important to have instrument overlap, to be able to track instrument stability, to quantify measurement uncertainties, and to establish an absolute measurement scale traceable to the International System of Units (SI). The Moderate Resolution Imaging Spectroradiometer (MODIS) is a key instrument for both the Terra and Aqua missions, which were launched in December 1999 and May 2002, respectively. It has 20 reflective solar bands (RSB) with wavelengths from 0.41 to $2.2 \mu \mathrm{m}$ and observes the Earth at three nadir spatial resolutions: $0.25 \mathrm{~km}, 0.5 \mathrm{~km}$, and $1 \mathrm{~km}$. MODIS RSB on-orbit calibration is reflectance based with reference to the bi-directional reflectance factor (BRF) of its on-board solar diffuser (SD). The SD BRF characterization was made pre-launch by the instrument vendor using reference samples traceable directly to the National Institute of Standards and Technology (NIST). On-orbit SD reflectance degradation is tracked by an on-board solar diffuser stability monitor (SDSM). This paper provides details of this calibration chain, from prelaunch to on-orbit operation, and associated uncertainty assessments. Using MODIS as an example, this paper also discusses challenges and key design requirements for future missions developed for accurate climate studies.
\end{abstract}

Keywords: MODIS, calibration, traceability, uncertainty, reflective solar bands, solar diffuser 